\title{
Average Linkage Cluster Analysis
}

National Cancer Institute

\section{Source}

National Cancer Institute. Average Linkage Cluster Analysis. NCI Thesaurus. Code C65183.

A clustering method that determines the mean distance between all possible inter- or intra-cluster pairs. 